\title{
A Brief Comment on Vasa Vasorum of Human Saphenous Vein: relevance for Coronary Artery Bypass Surgery
}

Andrzej Loesch ${ }^{1}$, PhD DSc; Michael Richard Dashwood², PhD

DOI: $10.21470 / 1678-9741-2020-0066$

\begin{abstract}
The importance of the vasa vasorum and blood supply to the wall of human saphenous vein (hSV) used for coronary artery bypass grafting (CABG) is briefly discussed. This is in the context of the possible physical link of the vasa vasorum connecting with
\end{abstract}

the lumen of $\mathrm{hSV}$ and the anti-ischaemic impact of this microvessel network in the hSV used for CABG.

Keywords: Saphenous Vein. Vasa Vasorum. Coronary Artery Bypass. Microvessels. Cardiovascular System. Femoral Vein.

\begin{tabular}{ll}
\hline \multicolumn{2}{l}{ Abbreviations, acronyms \& symbols } \\
\hline ar & $=$ Arterioles \\
CABG & $=$ Coronary artery bypass grafting \\
hSV & $=$ Human saphenous vein \\
NA & $=$ Noradrenaline \\
NT & $=$ No-touch \\
PVAT & $=$ Perivascular adipose tissue \\
SV & $=$ Saphenous vein \\
ve & $=$ Venule \\
\hline
\end{tabular}

\section{INTRODUCTION}

The importance of the vasa vasorum in the performance (patency) of human saphenous vein (hSV) used for coronary artery bypass grafting (CABG) has been highlighted in various publications over the last 15 years ${ }^{[1-9]}$. It has been pointed out that the vasa system not only plays a role in delivering oxygen and nutrients to the vein wall — as it is generally accepted but it also may act as a novel 'transport system ${ }^{[10]}$. Principally the vasa vessels, including those located in the adventitia and those associated with the vein's surrounding pedicle of fat ${ }^{[11]}$, protect the hSV once implanted as grafts during CABG. A prime focus of this mini-review is on the luminal aspect of hSV and a possible link, either via tributaries or by direct contact, with the vein's rich vasa vasorum (venarum) system as elegantly presented previously ${ }^{[3,4,12,13]}$.

\section{Are there Vasa Openings into the Saphenous Vein Lumen?}

One of the intriguing questions regarding blood supply through the wall of the hSV is whether there are direct openings (terminations) of the vasa vasorum into the vein lumen? This phenomenon was originally observed in the human femoral vein, examined with the use of a scanning electron microscope, by Brook, over 50 years ago ${ }^{[14]}$. Such observations indicated the possibility of physical communication between the vasa vasorum and the lumen of the host vein. However, according to Brook ${ }^{[14]}$, no such communication between the vasa microcirculation and the lumen was observed in the case of hSV. Similar observations and doubt in the existence of such direct vasa openings to hSV lumen have also been expressed and broadly discussed by Kachlik et al. ${ }^{[3]}$. Here, we revisit some of the previous observations and discussions in an attempt to clarify this topic.

Studies of hSV with India ink showed that the nearest/closest vasa vessels to the vein lumen are at a distance of $\sim 70-100 \mu \mathrm{m}$, suggesting that there are no direct vasa contacts and openings

Correspondence Address:

\section{Andrzej Loesch}

(iD) https://orcid.org/0000-0002-8051-0099

Centre for Rheumatology and Connective Tissue Diseases, Division of Medicine, University College London Medical School, Royal Free Campus

Rowland Hill Street, London, United Kingdom - Zip Code: NW3 2PF

E-mail:aloesch@talktalk.net, a.loesch@ucl.ac.uk 
in the hSV lumen ${ }^{[3]}$. Also, no evidence of vasa openings at the hSV lumen was observed in those vein segments affected by hyperplasia. In such cases, however, the India ink labelled some of the vasa vessels adjacent to subintimal regions associated with hyperplastic changes ${ }^{[3]}$. According to Fernández-Alfonso et al. ${ }^{[9]}$, when India ink is injected through the lumen of hSV, in vitro, it can subsequently be detected within the vein wall - in the medial and adventitial vasa vasorum - as well as within the vein-associated pedicle of perivascular adipose tissue (PVAT). Hence, these results clearly imply continuity between the vein lumen and these vascular structures labelled by the India ink. However, it is difficult, or perhaps even impossible, to identify any structural continuity between these India ink-labelled vasa structures, as observed in a given plane of a standard histological circumferential or longitudinal section of the vein. Therefore, regardless of whether the India ink is injected via the adventitial or luminal aspect of hSV (including the no-touch [NT] preparations of hSV for CABG, where adventitia and PVAT are left intact and the vein is not distended ${ }^{[15]}$ ), the course of the India ink travel from the lumen and dense vasa vasorum system might still be unclear. For more details on India ink-stained hSV vasa vasorum, see Kachlik et al. ${ }^{[3,4]}$ and Fernández-Alfonso et al. ${ }^{[9]}$.

\section{Corrosion Casts of the hSV Vasa Vasorum}

Vascular corrosion cast studies of hSV have revealed the structural complexity of the vein vasa vasorum system ${ }^{[3,4,12,13]}$. In these studies, which included hSV segments harvested for CABG as well as hSV specimens obtained after postmortem delay, no direct openings of vasa vessels to hSV lumen were reported. Kachlik et al. ${ }^{[3]}$ have argued that if such openings were indeed present, then signs of these structures (openings) would be visible in the vascular corrosion casts of luminal aspects of hSV; these would appear at least as "blind ending vessels with rounded tips" if the luminal openings of vasa were not completely filled by the cast resin. This is an important observation as the viscosity of cast resin is usually much higher than the viscosity of blood, so the vascular infiltration by cast resin could be minimal or none in some circumstances (e.g., during vasoconstriction or at other obstructions). One would not expect to get "normal" flow of 'viscous' resin through vasa vasorum/microvessels in postmortem samples as this tissue is, by definition, dead.

\section{Existence vs. Non-existence of Vasa Direct Openings}

Discussions regarding the existence vs. non-existence (or absence?) of direct openings of vasa at the hSV lumen are enriched by the results of elegant studies by Dr. Thomas $\mathrm{P}$ Crotty, who examined vasoregulatory function of the venous microcirculation using the canine lateral saphenous vein (SV), as well as skin flaps, as experimental models $s^{[8,16-20]}$. According

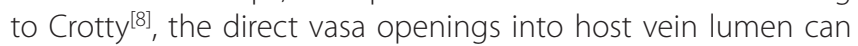
exist but are difficult to observe as they may be closed during smooth, non-turbulent, blood flow. In contrast, under turbulent flow or following stimulation with noradrenaline (NA), direct vasa openings can be seen at the intima of the host vein lumen. Such phenomena might explain, at least in part, the lack of findings of vasa openings (or "blind ending vessels") in corrosion

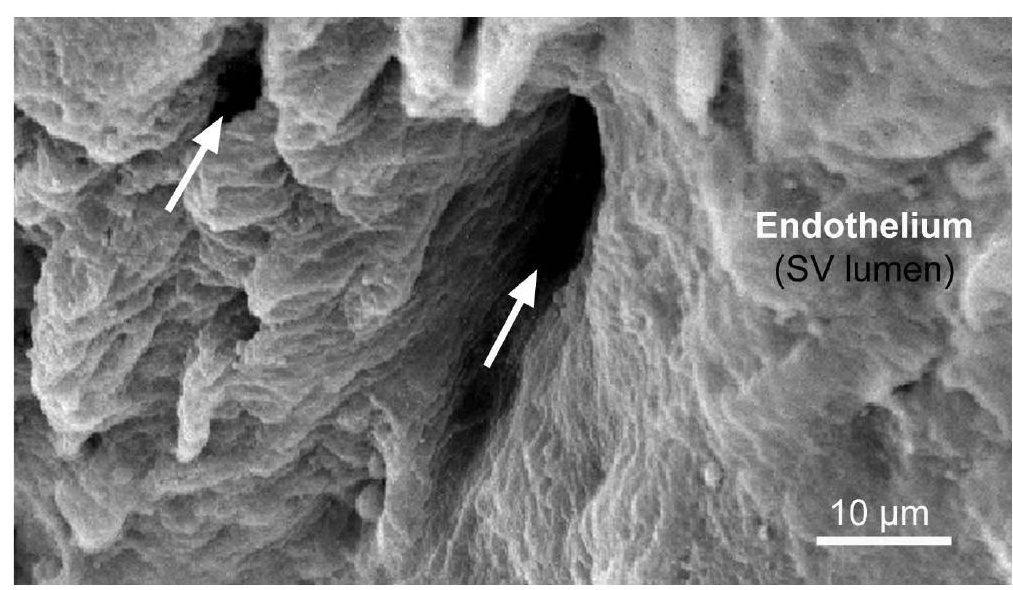

Fig. 1 - Scanning electron microscope (standard procedure) example of possible vasa vasorum/venarum direct openings into the lumen of the host vein - in this case of no-touch human saphenous vein (hSV) for coronary artery bypass grafting. Arrows point to the vasa openings of which one is $<10 \mu \mathrm{m}$ of diameter, while the other is $>10 \mu \mathrm{m}$. Also note that the arrows indicate a possible direction of blood flow - from the vein lumen into the openings of vasa to be then delivered to the vein wall following vein implantation as coronary graft. For more details on the aspects of blood flow in hSV as coronary graft, see Loesch and Dashwood ${ }^{[10]}, 2018$. SV $=$ saphenous vein

casts of $\mathrm{hSV} \mathrm{V}^{[3]}$, where the vasa was subjected to non-turbulent perfusion with rather high (higher than that of blood) viscosity resin. Nevertheless, and in agreement with Crotty ${ }^{[21]}$, one has to recognise the significant structural and pharmacological differences between canine lateral SV and hSV. Again, and regardless of these differences, observations of the so-called NT hSV harvested for CABG (where there is no vein stripping and distension applied) reveal retrograde blood flow from the graft lumen to the adventitial vasa circulation, and where small calibre vessel-like openings of $\sim 4$ to $6 \mu \mathrm{m}$ in diameter have been found in the graft luminal aspect ${ }^{[6]}$. The size of these vasa openings observed by Dreifaldt et al. ${ }^{[6]}$ therefore agrees with the report of vasa capillaries in hSV wall as small as $4.7 \mu \mathrm{m}$ to $11.6 \mu \mathrm{m}^{[3]}$. The observations by Dreifaldt et al. ${ }^{[6]}$ and Crotty $^{[8]}$ clearly imply a functional role of such openings of vasa in the retrograde blood flow to the vein wall; importantly, this phenomenon also applies to the NT hSV when used as coronary grafts ${ }^{[6]}$. Here, Figure 1 demonstrates possible vasa vasorum direct openings to the lumen of hSV preparation for CABG as observed with the scanning electron microscope. And Figure 2 diagrammatically shows the state and consequences of damaged vasa vasorum in hSV harvested conventionally (where stripping and distention are applied) and conversely, the preserved, undamaged, vasa vasorum in NT hSV preparations for CABG.

It is therefore highly likely that the bulk of blood flowing to the vasa system in NT hSV grafts for CABG is via the vein tributaries, which remain present but are ligated some distance from the vein stem. This implies that the vasa vasorum of intact 
hSV in the lower limb may or may not contribute to blood flow to the vein lumen (via direct openings), while in NT hSV grafts for CABG, the blood from the lumen of the vein flows both via tributaries and via direct vasa openings to further supply the vasa microcirculation and the wall of venous graft $^{[6]}$. In this scenario, tributaries and possible direct vasa terminations in NT hSV grafts for CABG now play a role of feeding vessels to local vasa vasorum ${ }^{[10]}$.

\section{How is this Happening: Vasa Openings and Turbulence?}

The existence of small-sized direct openings, possibly linking vasa with the host vein lumen, does not necessarily mean that blood flow may occur in either direction at any time, namely from the vasa network into the vein lumen or vice versa. According to Crotty ${ }^{[8]}$, one of the factors that may influence the direction of flow is that of turbulence. This is the case because turbulent blood flow (or blood turbulence) introduces radial components/ forces (e.g., vortices), without which blood flow from the vein to its vasa microcirculation may not be possible. In theory, these radial components therefore act as "biological mini pumps" that distribute blood from the vein to its microcirculation/ vasa ${ }^{[8]}$. Nevertheless, the previously mentioned retrograde flow and the direct connections between, e.g., hSV lumen and the vein vasa vessels are in question. One argument presented by Kachlik et al. ${ }^{[3]}$ against the existence of such connections is an observation of red blood cells within the lumen of small calibre vasa vessels in hSV harvested for CABG ${ }^{[6,23]}$; according to Kachlik et al. ${ }^{[3]}$, such blood cells should not be present there after vein distention with saline (usually at pressure $300 \mathrm{~mm} \mathrm{Hg}$ or more). However, the luminal pressure applied during saline distension (n.b., not a saline perfusion) is distributed radially and possibly equally along the axis of hSV harvested for CABG. Therefore, there is no particular "dominating" saline flow vector, but rather a constant pressure is inflicted on the intima. Consequently, there is no movement and/or removal (wash out) of red cells from small calibre vasa vessels. In effect, red cells may remain stationary within vasa of non-leaking hSV preparations for $C A B G$. It is important to stress that the stripping, and in particular high pressure distension, that is routinely applied to overcome spasm of conventionally harvested hSV for CABG

\section{No-touch saphenous vein}

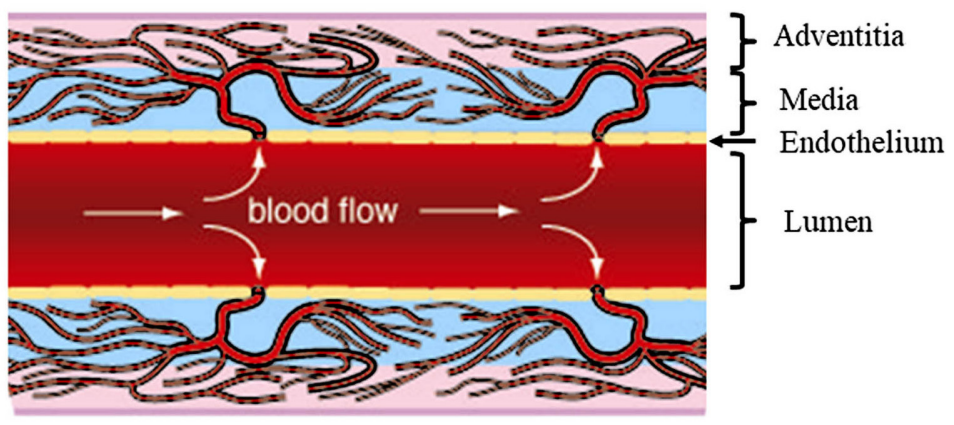

\section{Conventional saphenous vein}

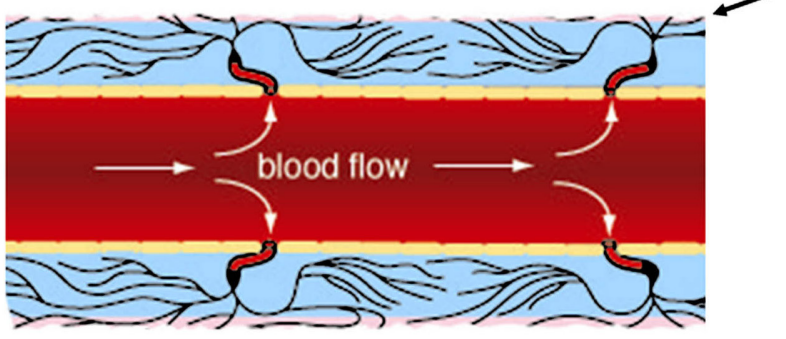

Adventitia damaged
Fig. 2 - Diagrammatically proposed reduction in blood flow in damaged vasa vasorum (medial 2015, and Loesch and Dashwood ${ }^{[10], 2018 . ~ F r o m ~ a n ~ O p e n ~ A c c e s s ~ a r t i c l e: ~ L o e s c h ~ a n d ~ D a s h w o o d ~[10], ~}$ 2018, which is acknowledged. causes distortion to the vein's original architecture, including the structure of the vein intima ${ }^{[15,23]}$. This may also distort and/or obscure direct small openings of vasa into the hSV lumen.

\section{Valve, Agger, Turbulence, and Vasa Vasorum}

Concerning the effects of turbulent flow, there is evidence of an impact of intact valves on the flow in reversed hSV used as infrainguinal vein grafts $^{[24]}$. This phenomenon also concerns hSV preparations for CABG, where valves (reversed) cause luminal narrowing, thus creating disturbances in blood flow ${ }^{[25-}$ 27]. It should be pointed out that hSV has numerous valves irregularly distributed throughout the vein length ${ }^{[28]}$. According to Portugal et al. ${ }^{[29]}$, the average number of valves in hSV (taken from the medial epicondyle of the femur to the saphenous hiatus) is around 4.77-4.87; with a range of two to nine valves being present, all of which were bicuspid. Within hSV valves, at the base of the valve sinus, is located a "bizarre" structure, the 


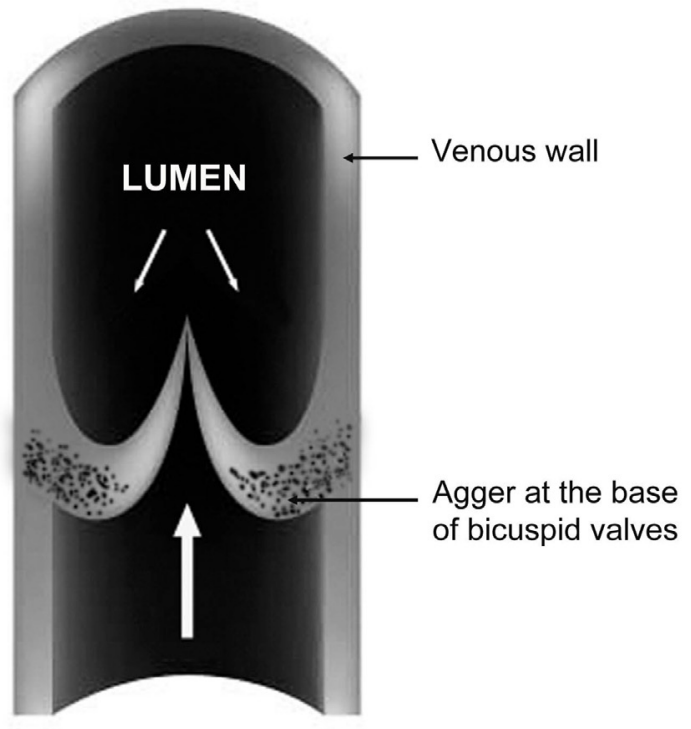

Fig. 3 - Diagrammatic representation of a longitudinal section through the vein (may be relevant to human saphenous vein) showing the venal bicuspid valves with their cusps/leaflets closed. Note that the agger is located at the base of valves. Large arrow indicates the main direction (against gravity) of blood movement, while the two small arrows, above valves, indicate a backflow of blood, in particular if valves are damaged in pathological conditions, and where the thickening of agger's structure (usually comprising smooth muscle and elastin) also occurs. For more details about venal valve and agger, see, e.g,. Corcos et al. ${ }^{[31]}, 2000$, and Crotty $^{[8]}, 2011$.

agger, comprised mainly of smooth muscle and elastin [20,30,31]. Here, Figure 3 schematically shows the agger's location in the venal wall. In pathological conditions, such as varicose hSV with prolapsed valves, the agger's structure is enlarged due to thickened bundles of smooth muscle and the fragmentation and dissociation of the elastin membrane ${ }^{[31]}$. Apart from contributing to the control of blood flow in the vein lumen, healthy valves seem to also contribute to the vasa vasorum microcirculation system. This claim is supported by the observation of vasa vasorum openings at the base of the valve sinus located at tributary junctions, at least in the canine lateral SV ${ }^{[8,18]}$. According to Crotty ${ }^{[19]}$, the agger's structure is made up of bundles of smooth muscle cells and fibroelastin, with this structure playing a dual role: (i) in the movement of the valve; and (ii) in regulating blood flow between the vasa vasorum system and the lumen of the parental vein. The importance of blood flow between the vasa vasorum system and the lumen of the parental vein (please see sections above) is rarely discussed, in particular in the context of valve and agger's function. An exception seems to be the meticulous findings described by Crotty ${ }^{[20,8]}$. Accordingly, the agger is a crescentic, fibroelastic structure internal to the layer of circular smooth muscles of the vein wall; both the base of the agger and its apex (apex is located at the base of the valve sinus) rest on bundles of longitudinal smooth muscle cells. Consequently, when the venous tone rises, it constricts smooth muscles bundles of valve's agger. In effect, the agger's smooth muscles are pulling in opposite directions and the elastic fibres become stretched, which opens the lumen of local vasa vasorum venules that drain into the vein lumen. As an example, this has been observed in elegant studies of NA-elevated tone of canine lateral SV with injected India-ink ${ }^{[17,18,20]}$. Due to the structural features, including thickness, the role of valvular agger in human veins of lower limbs may also be to prevent local dilation of the vein ${ }^{[32]}$. Interestingly, there appears to be a lack of attention regarding the role of the agger, including in the hSV, since there is no mention of this structure in medical textbooks, such as early editions of The Pathology and Surgery of the Veins of the Lower Limb $^{[33]}$ or recent editions of the Gray's Anatomy.

In this mini-review, we have not discussed additional phenomena influencing physiology of the vasa vasorum such as the impact of the rich supply of nerves, in particular the sympathetic innervation of the hSV ${ }^{[5]}$ (Figure 4). However, it is known from Crotty's elegant studies that sympathetic nerves have a regulatory function in relation to (i) venous vasa vasorum microcirculation; (ii) valve and agger's function; (iii) mediating and/or competing with plasma NA; and finally (iv) the origin of varicose veins ${ }^{[8]}$. The extent to which Dr. Crotty appreciated the influence of sympathetic nerves and/or NA become clear on reading the final sentence of the Abstract in his last paper ${ }^{[34]}$ : "Death is believed to occur when plasma noradrenaline has damaged the structure of the sympathetic system so much that it

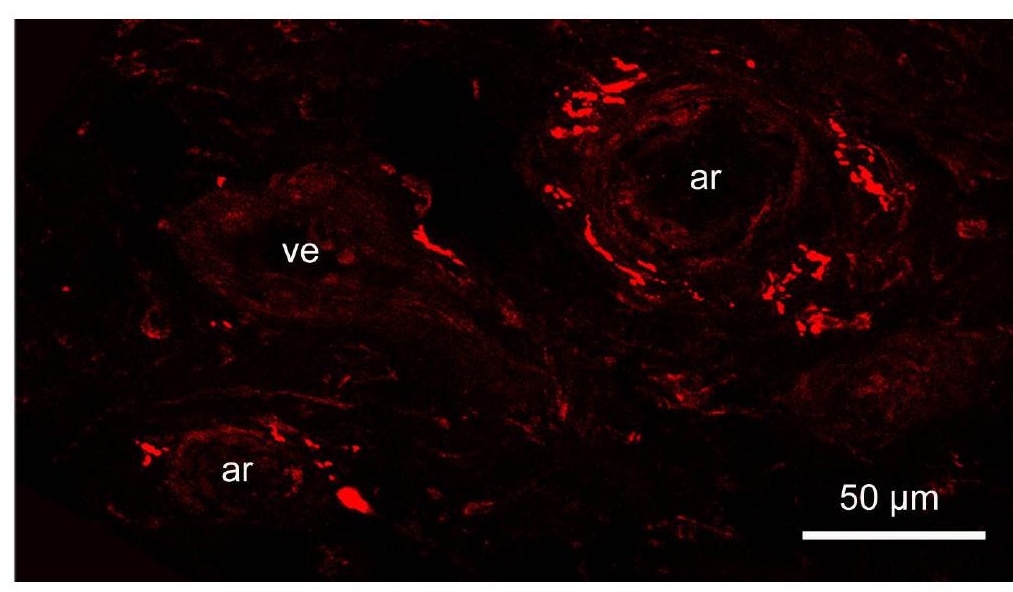

Fig. 4 - Confocal microscope image of no-touch human saphenous vein (hSV) (30 um frozen section) immunolabelled (originally red) for tyrosine hydroxylase ( $\mathrm{TH}$; a marker for sympathetic nerves). A fragment of adventitia periphery showing TH-positive perivascular sympathetic nerves at small vasa vasorum blood vessels: two arterioles (ar) and one venule (ve). Bar: $50 \mu \mathrm{m}$. Note that a rabbit TH polyclonal antibody (TZ 1010, Affinity, Exeter, United Kingdom) was used at 1:300; goat antirabbit Alexa 568 (Molecular Probes, Oregon, United States of America) was used at 1:600 as a second layer; confocal laser microscope: BioRadiance 2000. For more details on the sympathetic innervation of hSV, see Loesch and Dashwood 2009. 
can no longer create the minimum quantity of neurotransmitter needed to maintain the level of noradrenergic balance and homeostasis necessary for life."

\section{CONCLUSION}

Histological studies (e.g., using India ink, the pharmacology of NA, and those with scanning electron microscope) support the hypothesis that there are luminal openings/terminations of vasa in the lumen of hSV (NT hSV) used for CABG. Notably, the occurrence of blood flow through the vasa of NT hSV used as grafts during CABG is the strongest indication, yet that there is a direct link (physical communication) between the hSV lumen and the vein vasa vasorum microcirculation. This phenomenon may have a physiologically protective anti-ischemic effect on the graft, particularly in its early stages after implantation into the coronary circulation.

\section{ACKNOWLEDGEMENTS}

We gratefully acknowledge provision of hSV samples that were obtained with patients' consent and in accordance with the ethical approval from the Örebrö University Cardiothoracic Surgery, Örebrö, Sweden, and for this we thank Dr. Mats Dreifaldt and Dr. Domingos Souza.

\section{No financial support. \\ No conflict of interest.}

\section{Authors' roles \& responsibilities}

AL Substantial contributions to the conception or design of the work; drafting the work or revising it critically for important intellectual content; agreement to be accountable for all aspects of the work in ensuring that questions related to the accuracy or integrity of any part of the work are appropriately investigated and resolved; final approval of the version to be published

MRD Substantial contributions to the conception or design of the work; drafting the work or revising it critically for important intellectual content; agreement to be accountable for all aspects of the work in ensuring that questions related to the accuracy or integrity of any part of the work are appropriately investigated and resolved; final approval of the version to be published

\section{REFERENCES}

1. Dashwood MR, Anand R, Loesch A, Souza DS. Hypothesis: a potential role for the vasa vasorum in the maintenance of vein graft patency. Angiology. 2004;55(4):385-95. doi:10.1177/000331970405500405.

2. Dashwood M, Loesch A, Filbey D, Dreifaldt M, Souza D. Improved saphenous vein graft patency in coronary artery bypass patients: the contribution of an intact vasa vasorum. In: Lewis BA, Halon DA, Flugelman
MY, eds. New Horizons in Coronary Artery Disease. Medimond S. r. I., International Proceedings, Bologna: Monduzzi Editore. 2007;193-197.

3. Kachlik D, Baca V, Stingl J, Sosna B, Lametschwandtner A, Minnich B, et al. Architectonic arrangement of the vasa vasorum of the human great saphenous vein. JVasc Res. 2007;44(2):157-66. doi:10.1159/000099142.

4. Kachlík D, Stingl J, Sosna B, Straka Z, Lametschwandtner A, Minnich B, et al. Morphological features of vasa vasorum in pathologically changed human great saphenous vein and its tributaries. Vasa. 2008;37(2):127-36. doi:10.1024/0301-1526.37.2.127.

5. Loesch A, Dashwood MR. On the sympathetic innervation of the human greater saphenous vein: relevance to clinical practice. Curr Vasc Pharmacol. 2009;7(1):58-67. doi:10.2174/157016109787354150.

6. Dreifaldt M, Souza DS, Loesch A, Muddle JR, Karlsson MG, Filbey D, et al. The "no-touch" harvesting technique for vein grafts in coronary artery bypass surgery preserves an intact vasa vasorum. J Thorac Cardiovasc Surg. 2011;141(1):145-50. doi:10.1016/j.jtcvs.2010.02.005.

7. Dreifaldt M, Souza D, Bodin L, Shi-Wen X, Dooley A, Muddle J, et al. The vasa vasorum and associated endothelial nitric oxide synthase is more important for saphenous vein than arterial bypass grafts. Angiology. 2013;64(4):293-9. doi:10.1177/0003319712443729.

8. Crotty TP. Varicose veins are caused by segmental failures of the vasoregulatory role of the venous microcirculation, mediated by plasma norepinephrine. In: Nelson AE, ed. Varicose Veins: Symptoms, Causes and Treatments. New York: Nova Science Publishers; 2011. Chapter 1:1-58.

9. Fernandez-Alfonso MS, Souza DS, Dreifaldt M, Dashwood MR. Commentary: perivascular fat and improved vein graft patency in patients undergoing coronary artery bypass surgery. CurrVasc Pharmacol. 2016;14(4):308-12. doi:10.2174/1570161114666160513150444.

10. Loesch A, Dashwood MR. Vasa vasorum inside out/outside in communication: a potential role in the patency of saphenous vein coronary artery bypass grafts. J Cell Commun Signal. 2018;12(4):631-43. doi:10.1007/s12079-018-0483-1.

11. Fernández-Alfonso MS, Gil-Ortega M, Aranguez I, Souza D, Dreifaldt $\mathrm{M}$, Somoza B, et al. Role of PVAT in coronary atherosclerosis and vein graft patency: friend or foe? Br J Pharmacol. 2017;174(20):3561-72. doi:10.1111/bph.13734

12. Kachlík D, Lametschwandtner A, Rejmontová J, Stingl J, Vanek I. Vasa vasorum of the human great saphenous vein. Surg Radiol Anat. 2003;24(6):377-81. doi:10.1007/s00276-002-0067-9.

13. Lametschwandtner A, Minnich B, Kachlik D, Setina M, Stingl J. Threedimensional arrangement of the vasa vasorum in explanted segments of the aged human great saphenous vein: scanning electron microscopy and three-dimensional morphometry of vascular corrosion casts. Anat Rec A Discov Mol Cell Evol Biol. 2004;281 (2):1372-82. doi:10.1002/ ar.a.20098.

14. Brook WH. Vasa vasorum of veins in dog and man. Angiology. 1977;28(5):351-60. doi:10.1177/000331977702800507.

15. Souza D. A new no-touch preparation technique. Technical notes. Scand J Thorac Cardiovasc Surg. 1996;30(1):41-4. doi:10.3109/14017439609107239.

16. Crotty TP, Hall WJ, Sheehan JD. A study of perfused isolated dog saphenous vein. Ir J Med Sci. 1971;140(7):305-15. doi:10.1007/ bf02937775.

17. Crotty TP. Increased responsiveness of the canine lateral saphenous vein segment to noradrenaline when flow from its lumen to its vasa vasorum was obstructed. Ir J Med Sci. 1988;157(11):365.

18. Crotty TP. The path of retrograde flow from the lumen of the lateral saphenous vein of the dog to its vasa vasorum. Microvasc Res. 1989;37(1):119-22. doi:10.1016/0026-2862(89)90077-0.

19. Crotty TP. The corrupted feedback hypothesis. Med Hypotheses. 2003;61(5-6):605-16. doi:10.1016/s0306-9877(03)00243-3.

20. Crotty TP. The venous valve agger and plasma noradrenaline-mediated 
venodilator feedback. Phlebology. 2007;22(3):1 16-30. doi:10.1258/02 6835507780807293.

21. Crotty TP. The origin and the progression of varicose veins. Med Hypotheses. 1992;37(4):198-204. doi:10.1016/0306-9877(92)90188-i.

22. Samano N, Geijer H, Liden M, Fremes S, Bodin L, Souza D. The notouch saphenous vein for coronary artery bypass grafting maintains a patency, after 16 years, comparable to the left internal thoracic artery: a randomized trial. J Thorac Cardiovasc Surg. 2015;150(4):880-8. doi:10.1016/j.jtcvs.2015.07.027.

23. Ahmed SR, Johansson BL, Karlsson MG, Souza DS, Dashwood MR, Loesch A. Human saphenous vein and coronary bypass surgery: ultrastructural aspects of conventional and "no-touch" vein graft preparations. Histol Histopathol. 2004;19(2):421-33. doi:10.14670/HH-19.421.

24. Tullis MJ, Primozich J, Strandness DE Jr. Detection of "functional" valves in reversed saphenous vein bypass grafts: identification with duplex ultrasonography. J Vasc Surg. 1997;25(3):522-7. doi:10.1016/s07415214(97)70263-5.

25. McCaughan JJ Jr, Walsh DB, Edgcomb LP, Garrett HE. In vitro observations of greater saphenous vein valves during pulsatile and nonpulsatile flow and following lysis. J Vasc Surg. 1984;1 (2):356-61.

26. Ku DN, Klafta JM, Gewertz BL, Zarins CK. The contribution of valves to saphenous vein graft resistance. J Vasc Surg. 1987;6(3):274-9.

27. Walsh DB, Downing S, Ahmed SW, Wallace RB. Valvular obstruction of blood flow through saphenous veins. J Surg Res. 1987;42(1):39-42. doi:10.1016/0022-4804(87)90062-x.

28. Spray TL, Roberts WC. Changes in saphenous veins used as aortocoronary bypass grafts. Am Heart J. 1977;94(4):500-16. doi:10.1016/s00028703(77)80046-x.

29. Portugal IB, Ribeiro Ide L, Sousa-Rodrigues CF, Monte-Bispo RF, Rocha AC. Distribution of saphenous vein valves and its practical importance. Rev Bras Cir Cardiovasc. 2014;29(4):564-8. doi:10.5935/1678-9741.20140038.

30. Minkov M, Kanellaki-Kyparissi M, Marinov G, Koliakou K, Knyazhev V, Kovatchev D. Venous valve agger in non-varicose and varicose great saphenous vein - clinico-pathological considerations. Ann Proc Bulge Med Assoc. 1989;4:65-66.

31. Corcos L, De Anna D, Dini M, Macchi C, Ferrari PA, Dini S. Proximal long saphenous vein valves in primary venous insufficiency. J Mal Vasc. 2000;25(1):27-36.

32. Caggiati A. The venous valves of the lower limbs. Plebolymphology. 2013;20(2):87-95.

33. Dodd H, Cockett FB. The pathology and surgery of the veins of the lower limb. 2 ed. Edinburgh: Churchill Livingstone; 1956.

34. Crotty TP. Ageing is a process where the growth effect of neuronal noradrenaline changes progressively in favour of the flow mediated, neurodegenerative and inflammatory effect of plasma noradrenaline. Med Hypotheses. 2016;93:106-12. 